\title{
Digital Phase Detector based on Hilbert Transform
}

\author{
Shuai GUAN*, Yi WANG, Dingbang XIAO, Zhi-hua CHEN, Xuezhong WU, Qiang XU \\ Microsytem Lab \\ National University of Defense Technology, \\ ChangSha, Hunan, China \\ *E-mail: yeyuhan9@ hotmail.com \\ $+*$ Corresponding author
}

\begin{abstract}
Based on the Hilbert transform algorithm, a digital phase detector for sinusoidal signal is proposed. A half-band IIR filter implemented by the all-pass method approximates the Hilbert transform to realize the orthogonal decomposition of the signal. The Prosthaphaeresis formulas and Cordic algorithm are used to deal with the phase difference. The method can effectively avoid the influence of signal amplitude and frequency fluctuation on the phase detection results, and improve the precision of phase detector. The phase detector is designed and verified on Xilinx's Artix 7 FPGA. Experiments show that the phase detector error is $0.3 \%$.
\end{abstract}

Keywords-hilbert; phase detector; FPGA

\section{INTRODUCTION}

Phase-locked loop(PLL) as an important circuit module in the circuit system plays an important role [1]. Widely used in sensor's signal processing, wireless communication systems, high-speed interface and other fields. The phase detector(PD) as an important part of PLL, its phase accuracy and speed directly determine the performance of PLL, thereby affecting the performance of the entire system.

Hilbert transform is an important tool in signal analysis and processing, and is used to construct analytical signals in signal systems. Hilbert transform can decompose the signal into two mutually orthogonal signals without affecting the amplitude of its spectral components. This paper is the use of this feature to achieve the signal orthogonal decomposition, to lay the foundation for the signal processing following.

\section{REALIZATION THEORY OF PHASE DETECTOR}

The structure of phase detector(PD) is shown as Figure 1, The input signal can be converted into two orthogonal signals by Hilbert transform. For the transformation of the four-channel signal using the Prosthaphaeresis formulas as equations

(2), we can get $\left(A_{1} A_{2} \sin \theta, A_{1} A_{2} \cos \theta\right)$. The phase difference $\theta$ can be obtained by the Cordic algorithm's cartesian coordinates to the polar coordinate transformation [2].

Hilbert transform is a set of mathematical equations that reflect the relationship between the real and imaginary parts of the signal Fourier transform (or amplitude and phase). The ideal Hilbert transform is defined as a DTLTI system with frequency response as $\quad$ (3) [3].

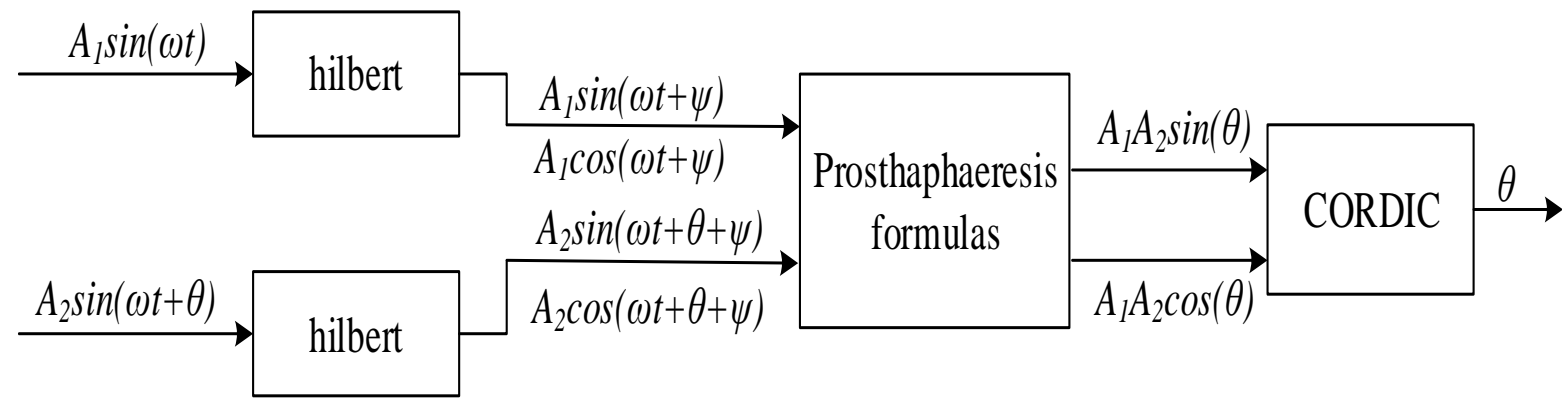

Figure 1. The structure of phase detector

$$
\begin{aligned}
A_{2} \sin (\omega t+\theta+\varphi) A_{1} \cos (\omega t+\varphi)- & A_{2} \cos (\omega t+\theta+\varphi) A_{1} \sin (\omega t+\varphi) \\
& =A_{1} A_{2} \sin (\omega t+\theta-\omega t) \\
& =A_{1} A_{2} \sin \theta
\end{aligned}
$$




$$
\begin{aligned}
& A_{2} \sin (\omega t+\theta+\varphi) A_{1} \sin (\omega t+\varphi)+A_{2} \cos (\omega t+\theta+\varphi) A_{1} \cos (\omega t+\varphi) \\
& =A_{1} A_{2} \cos (\omega t+\theta-\omega t) \\
& =A_{1} A_{2} \cos \theta \\
& H_{H T}\left(e^{j 2 \pi f}\right)=\left\{\begin{array}{cc}
-j, & 0 \leq f<\frac{1}{2} \\
j, & -\frac{1}{2} \leq f<0
\end{array}\right. \\
& \Delta_{p}=1-\left|H\left(e^{j 2 \pi F_{p}}\right)\right|^{2} \\
& \Delta_{s}=\left|H\left(e^{j 2 \pi F_{s}}\right)\right|^{2}
\end{aligned}
$$

Equation (3) can be derived by Fourier transform,

$$
H(n)=\left\{\begin{array}{cc}
0, & n \text { is even } \\
\frac{2}{n \pi}, & n \text { is odd }
\end{array}\right.
$$

Since the equation

(4) is not absolutely feasible, the ideal Hilbert transform is non-causal, so can't be achieved physical [4]. The Hilbert transform in engineering applications are implemented using function approximation methods. Common methods are using FIR, IIR and other methods to approximately. Compared to FIR filters, IIR can have fewer orders and better statistical characteristics, so this paper uses IIR filters to achieve Hilbert transform.

The main features of the Hilbert transform are,

$$
\begin{gathered}
F_{H p}=1-F_{H s} \\
\left|H_{H}\left(e^{j \pi}\right)\right|^{2}=\frac{1}{2} \\
\Delta_{H p}=\Delta_{H s} \Leftrightarrow a_{H p}=10 \log _{10}\left(1+\frac{1}{10^{a_{H s} / 10}-1}\right)
\end{gathered}
$$

Where,

$$
\begin{aligned}
& \Delta_{H p}=1-\left|H_{H}\left(e^{j 2 \pi F_{H p}}\right)\right|^{2} \\
& \Delta_{H s}=\left|H_{H}\left(e^{j 2 \pi F_{H s}}\right)\right|^{2}
\end{aligned}
$$

Considering the main features of the elliptical half band IIR filter are,

$$
\begin{gathered}
F=\frac{1}{2}-F_{s} \\
\left|H\left(e^{j \pi / 2}\right)\right|^{2}=\frac{1}{2} \\
\Delta_{p}=\Delta_{s} \Leftrightarrow a_{p}=10 \log _{10}\left(1+\frac{1}{10^{a_{s} / 10}-1}\right)
\end{gathered}
$$

Where,
From (5) and (7), we have,

$$
H_{H}(z)=H\left(z / e^{j \pi / 2}\right)=H(-j z)
$$

It can be seen from the equation (9) that the transfer function of the hilbert transform can be obtained by substituting $\mathrm{z}$ in the elliptic half band IIR filter transfer function $\mathrm{H}(\mathrm{z})$ with -jz. The full-pass implementation of the half-band IIR filter and the implementation of the hilbert converter are shown in and respectively.

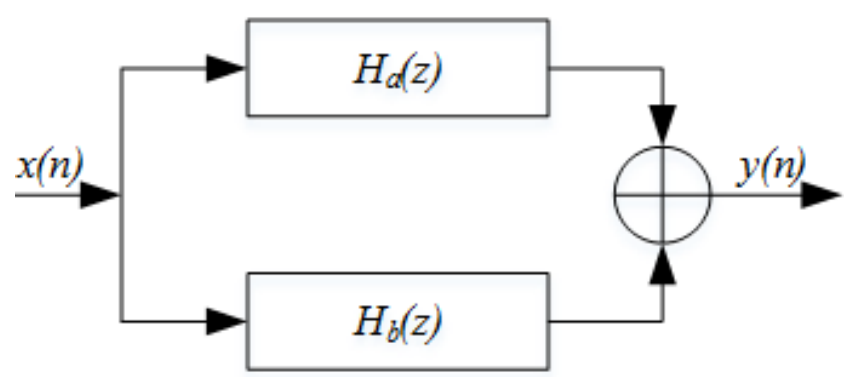

Figure 2. Realization of Half Band IIR Filter

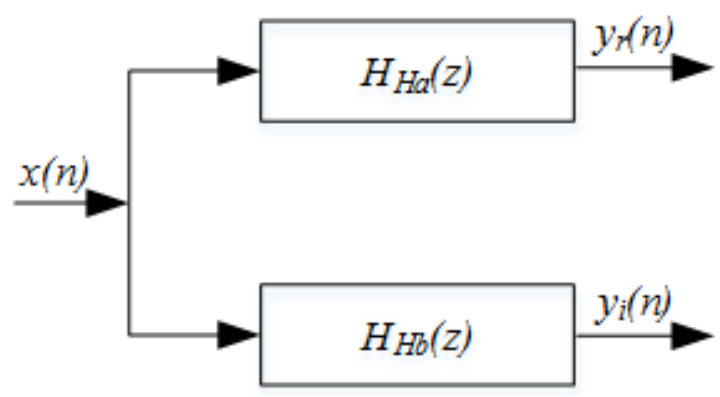

Figure 3. Realization of hilbert transform

The transfer function of IIR filter is,

$$
H(z)=\frac{1}{2}\left(H_{a}(z)+H_{b}(z)\right)
$$

Where, 


$$
\begin{aligned}
& H_{a}(z)=\prod_{i=[(n+7) / 4]}^{(n+1) / 2} \frac{\beta_{i}+z^{-2}}{1+\beta_{i} z^{-2}} \\
& H_{b}(z)=z^{-1} \prod_{i=3}^{[(n+1) / 4]} \frac{\beta_{i}+z^{-2}}{1+\beta_{i} z^{-2}}
\end{aligned}
$$

$$
H_{H}(z)=H_{H a}(z)+j H_{H b}(z)
$$

$$
\begin{aligned}
& H_{H a}(z)=\prod_{i=[(n+7) / 4]}^{(n+1) / 2} \frac{\beta_{i}-z^{-2}}{1-\beta_{i} z^{-2}}=\prod_{i=[(n+7) / 4]}^{(n+1) / 2} \frac{-\beta_{i}+z^{-2}}{1+\left(-\beta_{i}\right) z^{-2}} \\
& H_{H b}(z)=z^{-1} \prod_{i=3}^{[(n+1) / 4]} \frac{\beta_{i}+z^{-2}}{1+\beta_{i} z^{-2}}=(-1)^{(n+1) / 2} z^{-1} \prod_{i=3}^{[(n+1) / 4]} \frac{-\beta_{i}+z^{-2}}{1+\left(-\beta_{i}\right) z^{-2}}
\end{aligned}
$$

Comparing equations (11) and (13) can be found that the coefficient of the Hilbert converter is the coefficient of the negative half-band IIR filter. So the essence of the design Hilbert converter is consistent with the design of half band IIR filter.

In this paper, the design objective of the Hilbert converter is $\mathrm{SH}=\{\mathrm{FHs}, \mathrm{As}\}=\{0.53,46\}$, then the index of the half band IIR filter is $S=\{0.28,46\}$. When the order is $\mathrm{n}=9$, The filter coefficients are shown in TABLE I . Figure 4 shows the implementation of the hilbert converter.

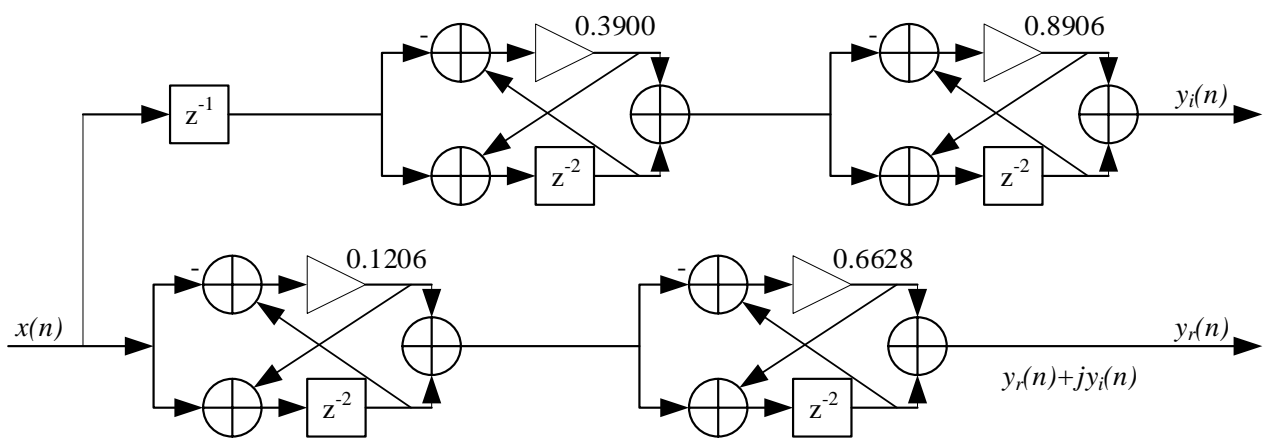

Figure 4. Hilbert transform implemented by 9-order elliptic complex IIR filter

\section{Model AND Simulation OF Phase Detector}

The hilbert transform simulation model in Simulink is shown as Figure 5. Figure 6.shows its bode. 


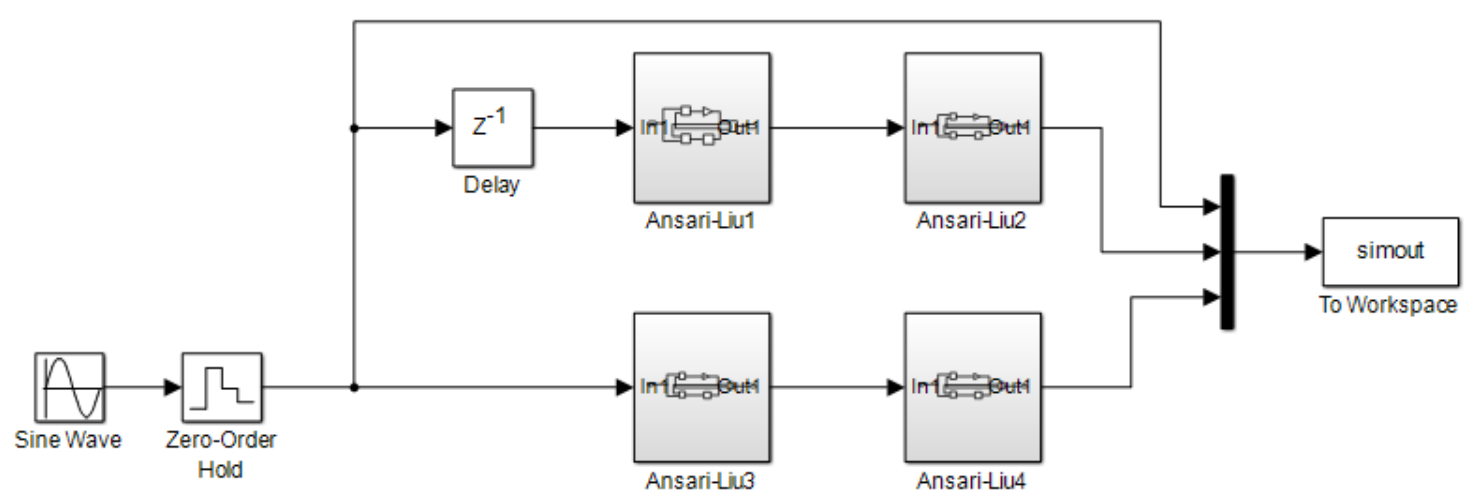

Figure 5. Simulation of hilbert transform

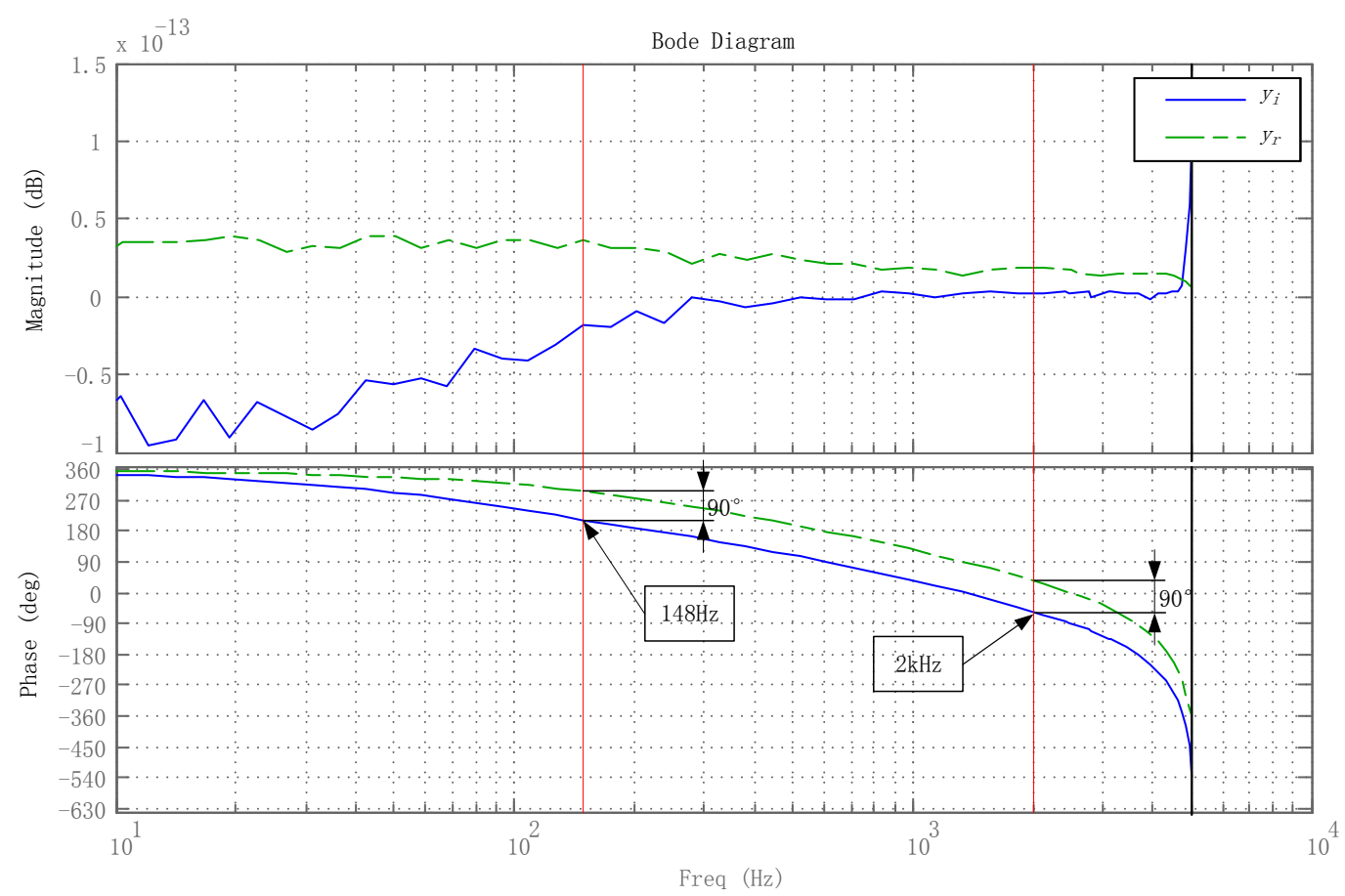

Figure 6. Bode diagram of hilbert transform

As can be seen from Figure 6, at a frequency between $148 \mathrm{~Hz}$ to $2 \mathrm{kHz}$, the output yi and yr phase difference is constant at $90^{\circ}$, and its amplitude gain is almost $0 \mathrm{~dB}$, so it can achieve $90^{\circ}$ phase shift function, and can't be affected by frequency within a certain range. The phase shift effect is shown in Figure 5, where yi is exactly $90^{\circ}$ from yr. Figure 6 shows the simulation of phase detector. Input two sinusoidal signal,

$$
x_{1}=\left\{\begin{array}{lc}
\sin (\omega t+0.3), & 0 \leq t<18 m s \\
\sin (\omega t+0.1), & t \geq 18 m s
\end{array}\right.
$$
$x_{2}=\sin (\omega t)$

, the response time is $2 \mathrm{~ms}$. After the system is stable, phase error is less than $0.15 \%$. 


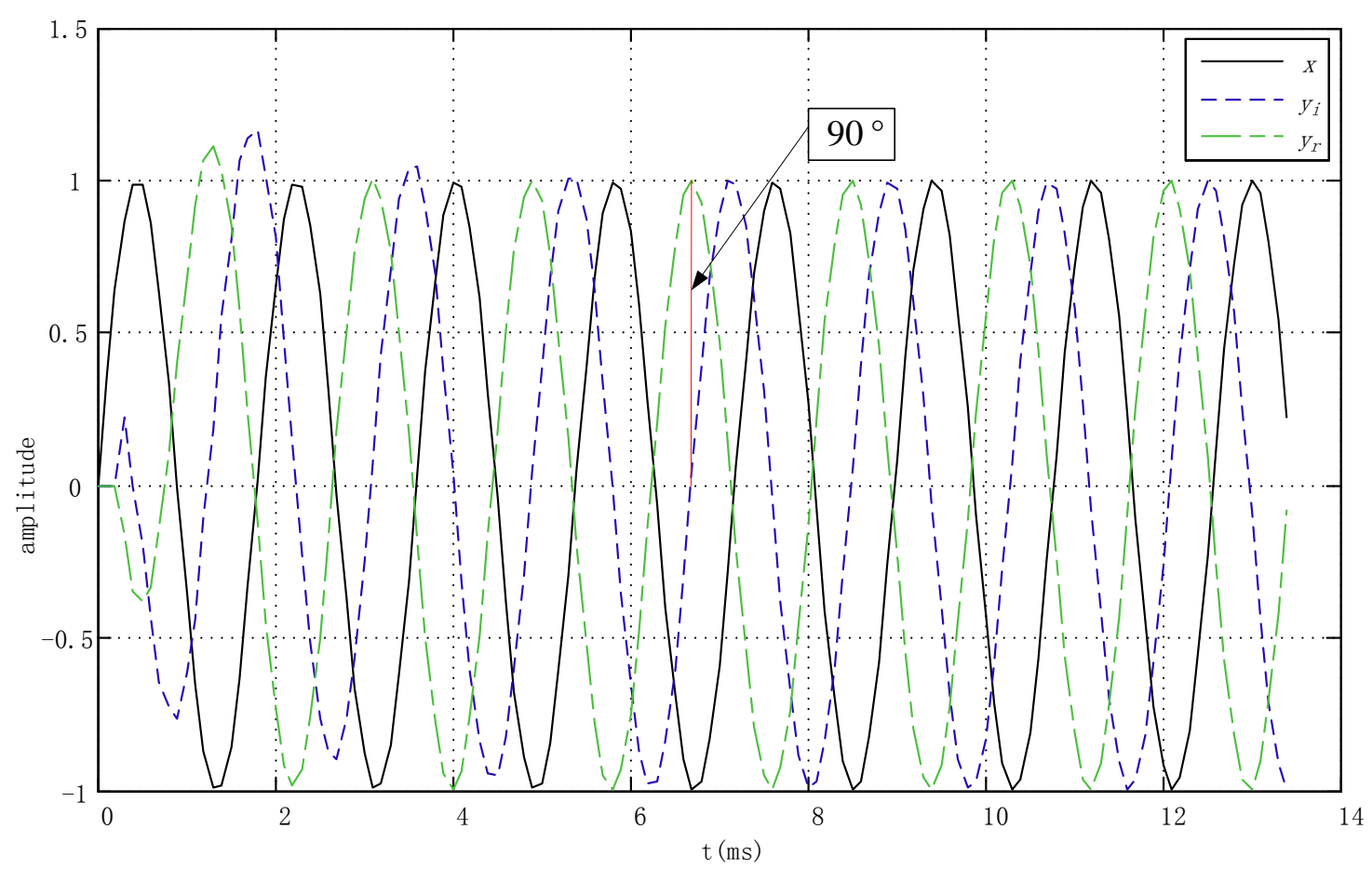

Figure 7. Hilbert transform on sine function

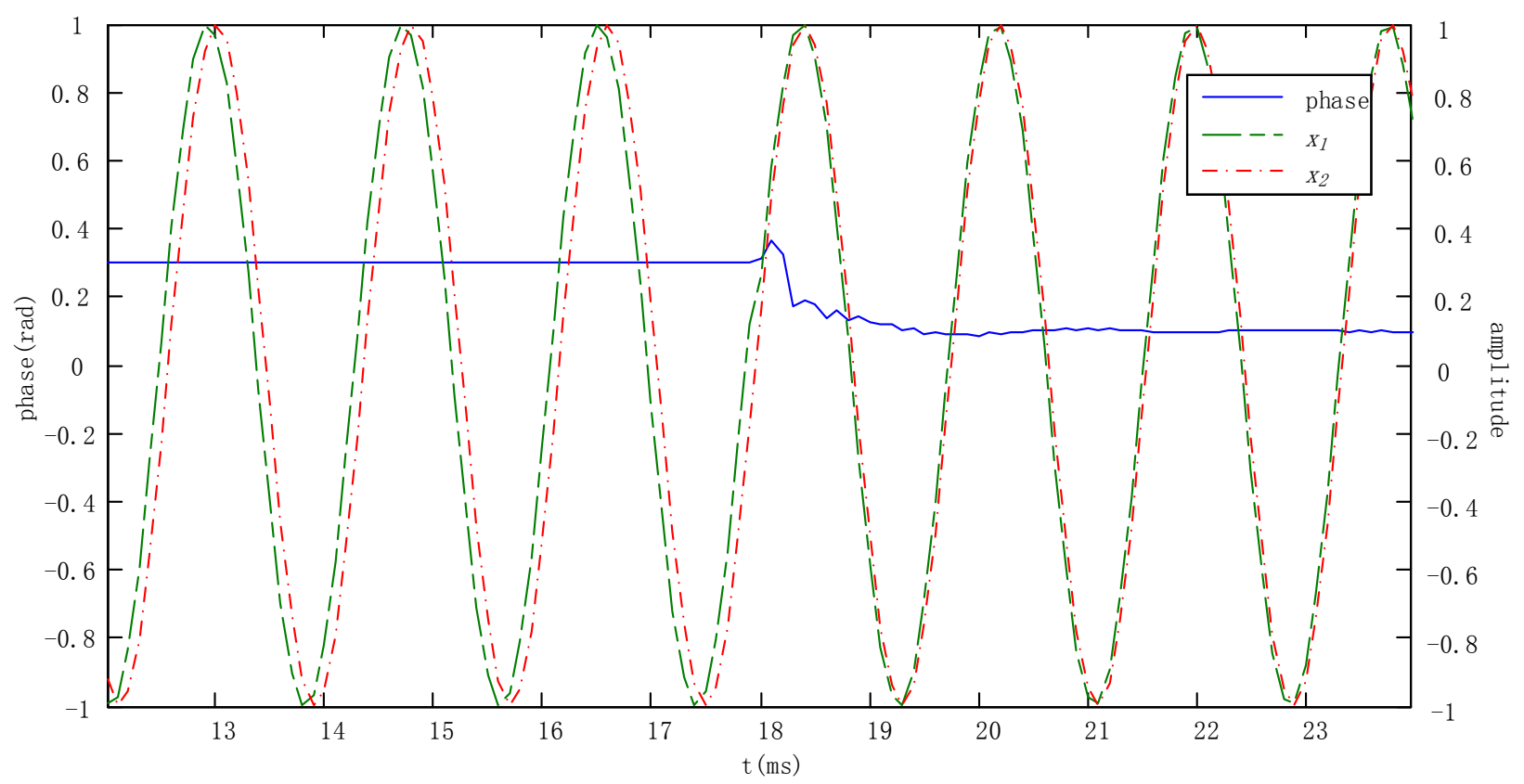

Figure 8. Simulation of phase detector

\section{DESIGN AND Test of Phase Detector On FPGA}

Using verilog hardware description language to achieve the design of the various modules in the modelsim in the test, and ultimately downloaded to the FPGA to verify the experiment.

\section{A. Hilbert Transform Test}

The coefficients in Table 1 were quantized by 11 bits. The results are shown in Table 2. The input data is 18 bits, taking into account the impact of the truncation error, the calculation uses 22 bits to ensure the output accuracy. Modelsim simulation shown in Figure 9. It can be seen from the figure, the converter output of the two signal's phase difference is $90^{\circ}$. Frequency is the same as input sinusoidal signal. 
TABLE II. QUANTIZATION TABLE OF HILBERT TRANSFORM COEFFICIENTS

\begin{tabular}{|c|c|c|}
\hline Coefficients $(\boldsymbol{\beta})$ & Hilbert transform & Hilbert converter quantization \\
\hline$\beta_{2}$ & 0.1206 & 00011110111 \\
\hline$\beta_{3}$ & 0.6628 & 10101001101 \\
\hline$\beta_{4}$ & 0.3900 & 01100011111 \\
\hline$\beta_{5}$ & 0.8906 & 11100100000 \\
\hline
\end{tabular}

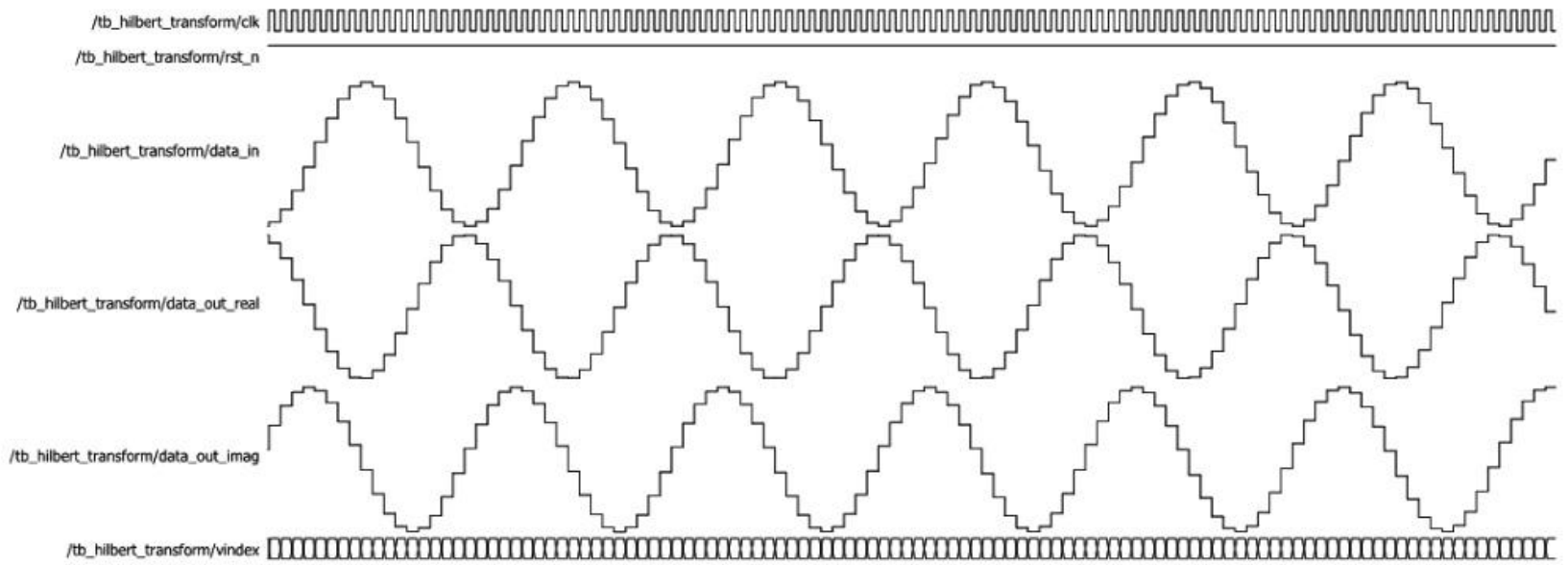

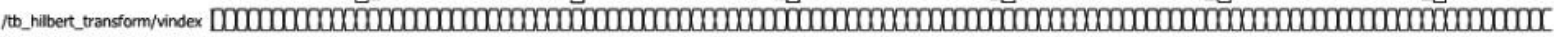

Figure 9. Hilbert transform timing simulation diagram

\section{B. Phase Detector Test}

Download the code into FPGA for intermodulation, using two signals which are created internal FPGA that have the same frequency and phase difference which is 0.3 .
The phase discrimination results of the phase detector are shown in Figure 10. Skewing is a 32-bit signal that converts the decimal fraction to 0.30132 . Phase error is $0.3 \%$, which can meet the requirements exactly.

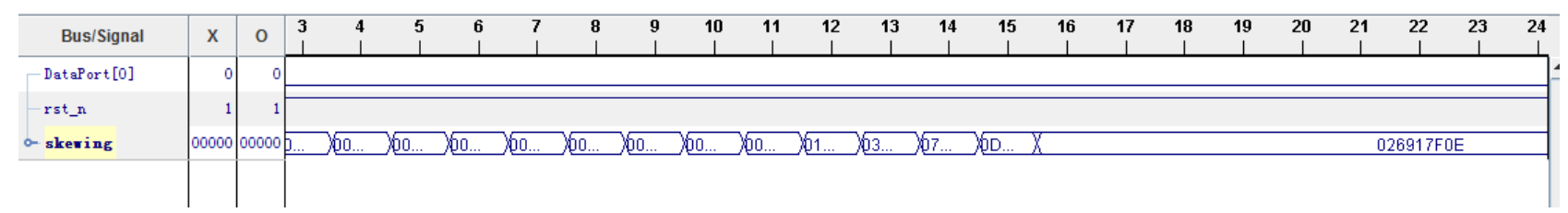

Figure 10. Result of phase detector

\section{CONCLUSIONS}

Based on the Hilbert transform algorithm, this paper presents a digital phase detector for sinusoidal signals. The phase detector system is designed and simulated in matlab, and using verilog hardware description language to achieve it. Finally, Xilinx's Artix7 FPGA is used as a platform to experiment and verify the design. The experimental results show that the phase detector can realize the phase discrimination of the sinusoidal signal, and the phase discrimination result is not affected by the amplitude fluctuation of the signal.

\section{REFERENCES}

[1] LIU Dan-dan, SHAN Chang-hong, SHENG Zhen and LI Feng-hua, "Research and design of a novel all digital phase locked loop working in broadband domain " Modern Electronics Technique, pp. 118-123, jan 2015.

[2] N Prasad, Ayas kanta Swain and K. K. Mahapatra, "FPGA Implementation of Pipelined CORDIC Based Quadrature Direct Digital Synthesizer with Improved SFDR," International Conference on Circuits, Power and Computing Technologies, pp. 756-760, 2013.

[3] Miroslav D. Lutovac, Dejan V. Tosic and Brian L. Evans, Filter Disign for Signal Processing Using MATLAB and Mathematica, Publicshing House of Electronics Industry, 2004.

[4] Meenakshi Aggarwal, Richa Barsainya and Tarun Kumar Rawat, "FPGA implementation of Hilbert transformer based on lattice wave digital filters," IEEE, 2015. 